\title{
Algebraic geometry informs perturbative quantum field theory
}

\author{
David Broadhurst* \\ Open University, UK, and Humboldt-Universität zu Berlin \\ E-mail: David.Broadhurst@open.ac.uk
}

\section{Oliver Schnetz}

University of Erlangen and Humboldt-Universität zu Berlin

E-mail: schnetz@mi.uni-erlangen.de

\begin{abstract}
Single-scale Feynman diagrams yield integrals that are periods, namely projective integrals of rational functions of Schwinger parameters. Algebraic geometry may therefore inform us of the types of number to which these integrals evaluate. We give examples at 3, 4 and 6 loops of massive Feynman diagrams that evaluate to Dirichlet $L$-series of modular forms and examples at 6,7 and 8 loops of counterterms that evaluate to multiple zeta values or polylogarithms of the sixth root of unity. At 8 loops and beyond, algebraic geometry informs us that polylogs are insufficient for the evaluation of terms in the beta-function of $\phi^{4}$ theory. Here, modular forms appear as obstructions to polylogarithmic evaluation.
\end{abstract}

In memoriam Jochem Fleischer (1937-2013)

Loops and Legs in Quantum Field Theory

27 April 2014 - 02 May 2014

Weimar, Germany

\footnotetext{
${ }^{*}$ Speaker.
} 


\section{Beyond polylogs: modular forms}

We begin with a short guide to modular forms. For $|q|<1$, let

$$
\eta(q) \equiv q^{1 / 24} \prod_{n>0}\left(1-q^{n}\right)=\sum_{n=-\infty}^{\infty}(-1)^{n} q^{(6 n+1)^{2} / 24}
$$

then for $\mathfrak{I} z>0$,

$$
\eta(\exp (2 \pi \mathrm{i} z))=(\mathrm{i} / z)^{1 / 2} \eta(\exp (-2 \pi \mathrm{i} / z)) .
$$

If $f(z)=(\sqrt{-N} / z)^{w} f(-N / z)$, we say that $f$ is a modular form of modular weight $w$ and level $N$. Here is a well known example with modular weight 12 and level 1:

$$
[\eta(q)]^{24}=\sum_{n>0} A(n) q^{n}=q-24 q^{2}+252 q^{3}-1472 q^{4}+4830 q^{5}-6048 q^{6}-16744 q^{7}+\ldots
$$

Its Fourier coefficients are multiplicative: $A(m n)=A(m) A(n)$ for $\operatorname{gcd}(m, n)=1$, and are determined by $A(p)$ at the primes $p$ :

$$
L(s) \equiv \sum_{n>0} \frac{A(n)}{n^{s}}=\prod_{p} \frac{1}{1-A(p) p^{-s}+p^{11-2 s}} .
$$

Moreover, we can analytically continue to values inside the critical strip:

$$
\Lambda(s) \equiv \frac{\Gamma(s)}{(2 \pi)^{s}} L(s)=\sum_{n>0} A(n) \int_{1}^{\infty} \mathrm{d} x\left(x^{s-1}+x^{11-s}\right) \exp (-2 \pi n x)=\Lambda(12-s) .
$$

\subsection{Multiplicative modular forms from eta-products}

For brevity, let $\eta_{n} \equiv \eta\left(q^{n}\right)$. Here are some multiplicative modular forms identified in quantum field theory

$\begin{array}{lccl}\text { form } & \text { weight level } \mathrm{QFT} \\ \eta_{1}^{3} \eta_{7}^{3} & 3 & 7 & \mathrm{BS} \\ \eta_{1}^{2} \eta_{2} \eta_{4} \eta_{8}^{2} & 3 & 8 & \mathrm{BS} \\ \eta_{2}^{3} \eta_{6}^{3} & 3 & 12 & \mathrm{BS}+\mathrm{BFT}+\mathrm{BBBG}+\mathrm{BV} \\ \eta_{1}^{4} \eta_{5}^{4} & 4 & 5 & \mathrm{BS} \\ \eta_{1}^{2} \eta_{2}^{2} \eta_{3}^{2} \eta_{6}^{2} & 4 & 6 & \mathrm{BS}+\mathrm{BB} \\ \eta_{1}^{4} \eta_{2}^{2} \eta_{4}^{4} & 5 & 4 & \mathrm{BS} \\ \eta_{1}^{6} \eta_{3}^{6} & 6 & 3 & \mathrm{BS} \\ \eta_{2}^{12} & 6 & 4 & \mathrm{BS} \\ \eta_{1}^{8} \eta_{2}^{8} & 8 & 2 & \mathrm{BS} \\ \eta_{1}^{24} & 12 & 1 & \mathrm{BK}\end{array}$

from work by Bailey, Borwein, Broadhurst, Glasser [1] (BBBG), Bloch, Vanhove [2] (BV), Broadhurst, Brown [7] (BB), Broadhurst, Fleischer, Tarasov [8] (BFT), Broadhurst, Kreimer [9, 10] (BK), Brown, Schnetz [11, 12] (BS).

Remark: QFT seems blind to Birch and Swinnerton-Dyer: nothing appears at weight 2. 


\section{Beyond polylogs and elliptic integrals}

Consider the two-loop massive sunrise diagram in $D=2$ spacetime dimensions:

$$
I\left(p^{2}, m_{1}, m_{2}, m_{3}\right) \equiv \frac{1}{\pi^{2}}\left(\prod_{k=1}^{3} \int \frac{\mathrm{d}^{2} q_{k}}{q_{k}^{2}-m_{k}^{2}+\mathrm{i} \varepsilon}\right) \boldsymbol{\delta}^{(2)}\left(p-q_{1}-q_{2}-q_{3}\right) .
$$

Following BBBG, we obtain an efficient result from the discontinuity across the cut [1]:

$$
I\left(w^{2}, m_{1}, m_{2}, m_{3}\right)=8 \pi \int_{m_{1}+m_{2}+m_{3}}^{\infty} \frac{A(x) x \mathrm{~d} x}{x^{2}-w^{2}}
$$

with an elliptic integral yielding the reciprocal

$$
A(w)=\frac{2}{\pi} \int_{0}^{\pi / 2} \frac{\mathrm{d} \theta}{\sqrt{F(w) \cos ^{2} \theta+16 m_{1} m_{2} m_{3} w \sin ^{2} \theta}}=\frac{1}{\operatorname{agm}(\sqrt{F(w)}, \sqrt{F(w)-F(-w)})}
$$

of an arithmetic-geometric mean with

$$
F(w)=\left(w+m_{1}+m_{2}+m_{3}\right)\left(w+m_{1}-m_{2}-m_{3}\right)\left(w-m_{1}+m_{2}-m_{3}\right)\left(w-m_{1}-m_{2}+m_{3}\right) .
$$

From the complementary elliptic integral

$$
B(w)=\frac{1}{\operatorname{agm}(\sqrt{F(w)}, \sqrt{F(-w)})}
$$

we obtain the elliptic nome

$$
q(w) \equiv \exp (-\pi B(w) / A(w)) .
$$

\subsection{Differential equation in the equal-mass case}

Now set $m_{1}=m_{2}=m_{3}=1$. Then $F(w)=(w+3)(w-1)^{3}$ and the differential equation, found in 1993 with Jochem Fleischer (sadly deceased on 2 April 2013) and Oleg Tarasov [8], gives

$$
-\left(\frac{q(w)}{q^{\prime}(w)} \frac{\mathrm{d}}{\mathrm{d} w}\right)^{2}\left(\frac{I\left(w^{2}, 1,1,1\right)}{24 \sqrt{3} A(w)}\right)=\frac{w^{2}\left(w^{2}-1\right)\left(w^{2}-9\right) A(w)^{3}}{9 \sqrt{3}} .
$$

Regarding $w$ and $A(w)$ as functions of $q$, we have a parametric solution

$$
\frac{w}{3}=\left(\frac{\eta_{3}}{\eta_{1}}\right)^{4}\left(\frac{\eta_{2}}{\eta_{6}}\right)^{2}, \quad 4 \sqrt{3} A=\frac{\eta_{1}^{6} \eta_{6}}{\eta_{2}^{3} \eta_{3}^{2}}
$$

Moreover, the two algebraic relations between $\left\{\eta_{1}, \eta_{2}, \eta_{3}, \eta_{6}\right\}$ give

$$
\frac{w^{2}-1}{8}=\left(\frac{\eta_{2}}{\eta_{1}}\right)^{9}\left(\frac{\eta_{3}}{\eta_{6}}\right)^{3}, \quad \frac{w^{2}-9}{72}=\left(\frac{\eta_{6}}{\eta_{1}}\right)^{5} \frac{\eta_{2}}{\eta_{3}} .
$$

Hence the BFT differential equation reduces to

$$
-\left(q \frac{\mathrm{d}}{\mathrm{d} q}\right)^{2}\left(\frac{I}{24 \sqrt{3} A}\right)=\frac{w}{3} f_{3,12}=\left(\frac{\eta_{3}^{3}}{\eta_{1}}\right)^{3}+\left(\frac{\eta_{6}^{3}}{\eta_{2}}\right)^{3}
$$

where, remarkably, $f_{3,12} \equiv\left(\eta_{2} \eta_{6}\right)^{3}$ is a weight-3 level-12 modular form found in massless $\phi^{4}$ theory by Brown and Schnetz at 9 loops [12]. 


\subsection{Bloch-Vanhove elliptic dilogarithm}

Define a character with $\chi(n)= \pm 1$ for $n= \pm 1 \bmod 6$ and $\chi(n)=0$ otherwise. Then

$$
-\left(q \frac{\mathrm{d}}{\mathrm{d} q}\right)^{2}\left(\frac{I}{24 \sqrt{3} A}\right)=\sum_{n>0} \frac{n^{2}\left(q^{n}-q^{5 n}\right)}{1-q^{6 n}}=\sum_{n>0} \sum_{k>0} n^{2} \chi(k) q^{n k} .
$$

Integrating twice and using the known imaginary part on the cut, we recover the BV result [2]

$$
\frac{I\left(w^{2}, 1,1,1\right)}{4 A(w)}=E_{2}(q)=-\pi \log (-q)-3 \sqrt{3} \sum_{k>0} \frac{\chi(k)}{k^{2}} \frac{1+q^{k}}{1-q^{k}}=-E_{2}(1 / q)
$$

with constants of integration that make $I$ finite at the pseudo-threshold, where $q=-1$.

\subsection{An elliptic trilogarithm at 3 loops}

The equal-mass three-loop sunrise integral $J(t)$ yields an elliptic trilogarithm for [3]

$$
\frac{2 J(t)}{\omega_{1}(t)}=E_{3}(q)=(-2 \log (q))^{3}+\sum_{k>0} \frac{\psi(k)}{k^{3}} \frac{1+q^{k}}{1-q^{k}}=-E_{3}(1 / q)
$$

with $\psi(k)=\psi(k+6)=\psi(-k), \psi(1)=-48, \psi(2)=720, \psi(3)=384, \psi(6)=-5760$ and

$$
q=\exp \left(-\frac{2 \pi}{3} \frac{A(\widetilde{w})}{B(\widetilde{w})}\right)
$$

where $A$ and $B$ are the elliptic integrals for the equal-mass two-loop case, but now evaluated at

$$
\widetilde{w}=\sqrt{1-t / 4}+\sqrt{4-t / 4}
$$

Then the transformation between the Green functions for hexagonal and diamond lattices given by BBBG in Eq. (188) of [1] provides $\omega_{1}(t)=(\widetilde{w} B(\widetilde{w}))^{2}$ as a solution to the homogeneous equation that is regular as $t \rightarrow \infty$. Underlying the expression for $q$ are the cubic and sesquiplicate modular transformations of [6]. The constants of integration for $E_{3}(q)$ are determined by the requirement that $J(t)$ is finite as $t \rightarrow 0$ and reproduces the value $J(0)=7 \zeta(3)$ proven by BBBG [1].

\section{Modular forms in massive QFT}

Here we consider multi-loop on-shell sunrise diagrams in two spacetime dimensions. At $L$ loops, these are given in coordinate space as single integrals over $N=L+2$ Bessel functions: $L+1$ copies of $K_{0}(x)$ from the internal lines and a single $I_{0}(x)$ from Fourier transformation with respect the external on-shell momentum. More generally, let

$$
S_{N, L} \equiv 2^{L} \int_{0}^{\infty} I_{0}(x)^{N-L-1} K_{0}(x)^{L+1} x \mathrm{~d} x .
$$

Then $S_{6,4}$ is indicative of some of the number theory entering $g-2$ at 4 loops, where Stefano Laporta is tackling diagrams with 5 fermions in the intermediate state. 
For convergence, we require that $L<N \leq 2 L+2$. With $N=2 L+2$ we require that $L>1$. BBBG proved that [1]

$$
\begin{gathered}
S_{1,0}=S_{2,1}=1, \quad S_{3,1}=\frac{2 \pi}{3 \sqrt{3}}, \quad S_{3,2}=\frac{4 \mathrm{Cl}_{2}(\pi / 3)}{\sqrt{3}}, \quad S_{4,2}=\frac{\pi^{2}}{4}, \quad S_{4,3}=7 \zeta(3), \\
S_{5,2}=\frac{\pi^{2}}{8}(\sqrt{15}-\sqrt{3})\left(\sum_{n=-\infty}^{\infty} \mathrm{e}^{-\sqrt{15} \pi n^{2}}\right)^{4}=\frac{\sqrt{3}}{120 \pi} \Gamma(1 / 15) \Gamma(2 / 15) \Gamma(4 / 15) \Gamma(8 / 15)
\end{gathered}
$$

where the final product of Gamma values results from the Chowla-Selberg theorem. York Schröder needed a counterterm in 3-dimensional lattice field theory[14] for which we used Chowla-Selberg to obtain the product $(\Gamma(1 / 24) \Gamma(11 / 24))^{2}$.

BBBG also conjectured (and checked to 1000 digits) that [1]

$$
S_{5,3}=\frac{4 \pi}{\sqrt{15}} S_{5,2}, \quad S_{6,4}=\frac{4 \pi^{2}}{3} S_{6,2}, \quad S_{8,5}=\frac{18 \pi^{2}}{7} S_{8,3} .
$$

Remark: In 4-dimensions, there are two master on-shell 3-loop sunrise integrals Amazingly, the finite parts of their Laurent expansions are linear combinations of $S_{5,3}$ and $\pi^{6} / S_{5,3}$, with the reciprocal coming from $\Gamma(7 / 15) \Gamma(11 / 15) \Gamma(13 / 15) \Gamma(14 / 15)$.

\subsection{Sunrise at 3 loops from a modular form of weight 3}

Let $L_{3,15}(s)$ be the Dirichlet $L$-function defined by the multiplicative modular form

$$
f_{3,15}=\left(\eta_{3} \eta_{5}\right)^{3}+\left(\eta_{1} \eta_{15}\right)^{3}
$$

with weight 3 and level 15. Then we conjecture (and have checked to 1000 digits) that [7]

$$
S_{5,2}=3 L_{3,15}(2), \quad S_{5,3}=\frac{8 \pi^{2}}{15} L_{3,15}(1),
$$

where $S_{5,3}$ is the 5-Bessel moment giving the on-shell 3-loop sunrise diagram.

\subsection{Sunrise at 4 loops from a modular form of weight 4}

Let $L_{4,6}(s)$ be the Dirichlet $L$-function defined by the multiplicative modular form

$$
f_{4,6}=\left(\eta_{1} \eta_{2} \eta_{3} \eta_{6}\right)^{2}
$$

with weight 4 and level 6 . Then we conjecture (and have checked to 1000 digits) that [7]

$$
S_{6,2}=6 L_{4,6}(2), \quad S_{6,3}=12 L_{4,6}(3), \quad S_{6,4}=8 \pi^{2} L_{4,6}(2),
$$

where $S_{6,4}$ is the 6-Bessel moment giving the on-shell 4-loop sunrise diagram.

\subsection{Almost sunrise at 6 loops from a modular form of weight 6}

Let $L_{6,6}(s)$ be the Dirichlet $L$-function defined by the multiplicative modular form

$$
f_{6,6}=\left(\frac{\eta_{2}^{3} \eta_{3}^{3}}{\eta_{1} \eta_{6}}\right)^{3}+\left(\frac{\eta_{1}^{3} \eta_{6}^{3}}{\eta_{2} \eta_{3}}\right)^{3}
$$

with weight 6 and level 6 . Then we conjecture (and have checked to 1000 digits) that [7]

$$
S_{8,3}=8 L_{6,6}(3), \quad S_{8,4}=36 L_{4,6}(4), \quad S_{8,5}=216 L_{4,6}(5),
$$

but have no result for $S_{8,6}$, the 8-Bessel moment for the on-shell 6-loop sunrise diagram. 


\section{Counterterms at 6 to 10 loops}

\subsection{6 loops: the first MZV}

In 1995, Broadhurst and Kreimer evaluated all the counterterms for the coupling of $\phi^{4}$ theory coming from subdivergence-free diagrams up to 6 loops [9]. All were expressible in terms of $\zeta(3)$, $\zeta(5), \zeta(7), \zeta(9)$ and $\zeta(5,3)-\frac{29}{12} \zeta(8)$, where the multiple zeta value $\zeta(5,3)=\sum_{m>n>0} m^{-5} n^{-3}$ is not reducible to single zeta values and occurs at 6 loops.

\subsection{7 loops: unexpected products}

At 7 loops, $\zeta(7,3)-\frac{793}{94} \zeta(10), \zeta(11)$ and $\zeta(3,5,3)-\zeta(3) \zeta(5,3)$ appear [9, 10]. In 1995, three 7-loop counterterms were lacking: $P_{7,8}, P_{7,9}$ and $P_{7,11}$ in the Schnetz census [18]. Allowing for a product of $\zeta(3)$ with a 6-loop counterterm, we eventually determined

$$
\begin{aligned}
P_{7,8}= & \frac{22383}{20} \zeta(11)+\frac{4572}{5}[\zeta(3,5,3)-\zeta(3) \zeta(5,3)]-700 \zeta(3)^{2} \zeta(5) \\
& +1792 \zeta(3)\left[\frac{9}{320}[12 \zeta(5,3)-29 \zeta(8)]+\frac{45}{64} \zeta(5) \zeta(3)\right], \\
P_{7,9}= & \frac{92943}{160} \zeta(11)+\frac{3381}{20}[\zeta(3,5,3)-\zeta(3) \zeta(5,3)]-\frac{1155}{4} \zeta(3)^{2} \zeta(5) \\
& +896 \zeta(3)\left[\frac{9}{320}[12 \zeta(5,3)-29 \zeta(8)]+\frac{45}{64} \zeta(5) \zeta(3)\right] .
\end{aligned}
$$

\subsection{7 loops: failures of the Kontsevich conjecture}

A sub-divergence free $\phi^{4}$ counterterm is of interest to algebraic geometers who regard it as a rather special type of "period". At 7 loops, with 14 edges, we have an integral over 13 Schwinger parameters:

$$
P_{G}=\int_{\alpha_{i}>0} \frac{\mathrm{d} \alpha_{2} \ldots \mathrm{d} \alpha_{14}}{\left|\Psi_{G}\left(\alpha_{1}, \alpha_{2}, \ldots, \alpha_{14}\right)\right|_{\alpha_{1}=1}^{2}}
$$

where physicists call $\Psi_{G}$ the first Symanzik polynomial and graph theorists call it the Kirchhoff polynomial of the graph $G$. Suppose that we count the number $c(q)$ of solutions to $\Psi_{G}=0$ in finite fields $\mathbf{F}_{q}$ for prime powers $q=p^{k}$. If $c(q)$ is a polynomial in $q$, then algebraic geometers expect the period $P_{G}$ to evaluate to MZVs.

Maxim Kontsevich conjectured that $c(q)$ is a polynomial for every graph $G$. This was proved true by Stembridge, by exhaustion, for all graphs with no more than 12 edges [21]. It is true for all 7-loop periods except [19] $P_{7,8}, P_{7,9}$ and $P_{7,11}$.

It is the prime $p=2$ that makes the periods $P_{7,8}$ and $P_{7,9}$ non-Stembridge $[15,19]$. Thus they were expected to be reducible to alternating sums in the MZV datamine [4]. Algorithms due to Panzer and Schnetz have achieved this. In each case a complicated combination of alternating sums collapses to MZVs, using the datamine.

\subsection{7 loops: Erik Panzer's polylogs of the sixth root of unity}

For the period $P_{7,11}$ the Stembridge count $c(p)$ takes different forms according as the residue of $p$ mod 3 [19] as may be quickly determined using the $c_{2}$ invariant of [13]. So we expect a reduction 
to weight- 11 multiple polylogs of the 6 th root of unity. There are $6^{2} \times 7^{9}=1,452,729,852$ of these with legal words.

Erik Panzer recently reported [17] a reduction of $P_{7,11}$ to a subset of these words and hence obtained several thousand digits of $P_{7,11}$, completing the 7-loop challenge. We have checked this answer to 21 digits, by intensive numerical methods. A compact presentation of Panzer's lengthy result has been obtained by Schnetz, using algebraic methods based on a co-action for polylogarithms. Moreover, Panzer was able to use PSLQ to reduce his result to a basis of dimension 72, resulting from applying the generalized parity conjecture of [5] to a three-letter Deligne alphabet.

\subsection{8 loops: planar $N=4$ supersymmetric Yang-Mills theory}

Without evaluating Feynman diagrams, Sébastien Leurent and Dmytro Volin have computed the Konishi anomalous dimension of planar $N=4$ SYM up to 8 loops [16]. An MZV first appears at 8 loops, where the weight- 11 term

$$
\frac{864 g^{16}}{5}\left\{76307 \zeta(11)+792[\zeta(3,5,3)-\zeta(3) \zeta(5,3)]-18840 \zeta(3)^{2} \zeta(5)\right\}
$$

is happily reducible to the 3 terms found in $\phi^{4}$ theory almost 20 years ago $[9,10]$.

Open question: Do MZVs suffice for the next "wrapping" at 12 loops?

\subsection{8 loops: further reductions to polylogs}

There are 41 targets in the census at 8 loops [18]. Thanks to recent progress with graphical functions [20] 31 of these have been evaluated in terms of polylogs. All periods for which weightdrop was predicted have been reduced to MZVs of weight less than 13. One period, $P_{8,16}$, exhibits weight mixing, evaluating to a mixture of weight-10 and weight-11 MZVs. Schnetz has evaluated the period $P_{8,33}$ as a compact combination of weight- 13 polylogs of the 6th root of unity. Of the 10 periods so far unidentified at 8 loops, it is anticipated that 6 may eventually be evaluated in terms of MZVs and polylogs of 4th or 6th roots of unity.

\subsection{8,9 and 10 loops: modular obstructions to polylogs}

Brown and Schnetz [12] have identified many periods with more than 7 loops for which there is strong evidence that polylogs cannot suffice for evaluations. There is no chain of integration over Schwinger parameters that satisfies the criterion of "linear independence" explained in [17]. In 16 cases, point counts in finite fields of the non-linear denominators correspond, modulo primes, to the Fourier coefficients of well known modular forms. In particular $\left(\eta_{1} \eta_{7}\right)^{3},\left(\eta_{1} \eta_{5}\right)^{4},\left(\eta_{1} \eta_{8}\right)^{2} \eta_{2} \eta_{4}$ and $\left(\eta_{1} \eta_{3}\right)^{6}$ are the modular forms that prevent reduction to polylogs of 4 subdivergence-free diagrams that give scheme-independent contributions to the 8-loop beta-function of $\phi^{4}$ theory. For these, one may need a theory of "multiple modular values" whose details are at present obscure.

Acknowledgements: We thank our colleagues Spencer Bloch, Francis Brown, Dzmitry Doryn, Dirk Kreimer, Erik Panzer and Karen Yeats for many illuminating discussions of the periods of quantum field theory and their relationship to algebraic geometry. 


\section{References}

[1] D.H. Bailey, J. M. Borwein, D. Broadhurst, M. L. Glasser, Elliptic Integral Evaluations of Bessel Moments, J. Phys. A41 (2008) 205203 [arXiv: 0801 . 0891 ].

[2] S. Bloch, P. Vanhove, The elliptic dilogarithm for the sunset graph [arXiv:1309.5865].

[3] S. Bloch, M. Kerr, P. Vanhove, A Feynman integral via higher normal functions [arXiv:1406.2664].

[4] J. Blümlein, D. J. Broadhurst, J. A. M. Vermaseren, The Multiple Zeta Value data mine, Comput. Phys. Commun. 181 (2010) 582-625, [arXiv: 0907 .2557].

[5] D.J. Broadhurst, Massive 3-loop Feynman diagrams reducible to SC* primitives of algebras of the sixth root of unity, Eur. Phys. J. C8 (1999) 311-333 [arXiv: hep-th/9803091].

[6] D. Broadhurst, Elliptic integral evaluation of a Bessel moment by contour integration of a lattice Green function [arXiv:0801.4813].

[7] D. Broadhurst, Multiple Zeta Values and Modular Forms in Quantum Field Theory in Computer Algebra in Quantum Field Theory 33-72, edited by C. Schneider and J. Blümlein (Springer) 2013.

[8] D.J. Broadhurst, J. Fleischer, O.V. Tarasov, Two-loop two-point functions with masses: asymptotic expansions and Taylor series, in any dimension, Z. Phys. C60 (1993) 287-302 [arXiv:hep-ph/9304303].

[9] D.J. Broadhurst, D. Kreimer, Knots and numbers in $\phi^{4}$ theory to 7 loops and beyond, Int. J. Mod. Phys. C6 (1995) 519-524 [arXiv: hep-ph/9504352].

[10] D.J. Broadhurst, D. Kreimer, Association of multiple zeta values with positive knots via Feynman diagrams up to 9 loops, Phys. Lett. B393 (1997) 403-412 [arXiv : hep-th/9609128].

[11] F. Brown, O. Schnetz, A K3 in $\phi^{4}$, Duke Math. J. 161 (2012) 1817-1862 [arXiv: 1006.4064 ].

[12] F. Brown, O. Schnetz, Modular forms in Quantum Field Theory, Commun. Number Theory Phys. 7 (2013) 293-325 [arXiv: 1304 .5342].

[13] F. Brown, O. Schnetz, K. Yeats, Properties of $c_{2}$ invariants of Feynman graphs [arXiv:1203.0188]

[14] F. Di Renzo, M. Laine, Y. Schröder, C. Torrero, Four-loop lattice-regularized vacuum energy density of the three-dimensional SU(3) + adjoint Higgs theory, JHEP 9 (2008) 061 [arXiv: 0808 . 0557].

[15] D. Doryn, On one example and one counterexample in counting rational points on graph hypersurfaces, Lett. Math. Phys. 97 (2011) 303-315 [arXiv: 1006.3533$].$

[16] S. Leurent, D. Volin, Multiple zeta functions and double wrapping in planar $N=4$ SYM, Nuclear Physics B875 (2013) 757-789 [arXiv: 1302 . 1135].

[17] E. Panzer, Feynman integrals via hyperlogarithms [arXiv:1407.0074].

[18] O. Schnetz, Quantum periods: A census of $\phi^{4}$-transcendentals, Commun. Number Theory Phys. 4 (2010) 1-48 [arXiv:0801.2856].

[19] O. Schnetz, Quantum field theory over $\mathbf{F}_{q}$, Electron. J. Combin. 18 (2011) 102 [arXiv:0909.0905].

[20] O. Schnetz, Graphical functions and single-valued multiple polylogarithms [arXiv:1302.6445].

[21] J. Stembridge, Counting points on varieties over finite fields related to a conjecture of Kontsevich, Ann. Combin. 2 (1998) 365-385. 